\title{
METHOD OF MATCHED EXPANSIONS \& THE SINGULARITY STRUCTURE OF THE GREEN FUNCTION
}

\author{
MARC CASALS ${ }^{1}$, SAM DOLAN $^{2}$, ADRIAN C. OTTEWILL $^{3}$ and BARRY WARDELL 4 \\ ${ }^{1}$ School of Mathematical Sciences, Dublin City University, Glasnevin, Dublin 9, Ireland. E-mail: \\ marc.casals@dcu.ie \\ ${ }^{2}$ School of Mathematics, University of Southampton, University Road, Southampton, SO17 1BJ, \\ United Kingdom.E-mail: s.dolan@soton.ac.uk \\ ${ }^{3}$ Complex and Adaptive Systems Laboratory and School of Mathematical Sciences, University \\ College Dublin, Belfield, Dublin 4, Ireland. E-mail: adrian.ottewill@ucd.ie \\ ${ }^{4}$ Max-Planck-Institut für Gravitationsphysik, Albert-Einstein-Institut, 14476 Golm, Germany. \\ E-mail: barry.wardell@aei.mpg.de
}

\begin{abstract}
We present the first successful application of the method of Matched Expansions for the calculation of the self-force on a point particle in a curved spacetime. We investigate the case of a scalar charge in the Nariai spacetime, which serves as a toy model for a point mass moving in the Schwarzschild black hole background. We discuss the singularity structure of the Green function beyond the normal neighbourhood and the interesting effect of caustics on null wave propagation.
\end{abstract}

Keywords: Self-Force; Green Function; Caustics

\section{Introduction}

Extreme Mass Ratio Inspirals, such as a stellar-mass compact object orbiting around a supermassive black hole, can be understood within perturbation theory of General Relativity as the smaller body's motion deviating from the background geodesic due to a self-force. ${ }^{1}$ The calculation of the self-force is necessary in order to model the orbital motion and obtain accurate gravitational-wave templates. The expression for the self-force in a general spacetime is formally similar for the cases of an orbiting point scalar charge, electrical charge and a point mass. In all these cases, the selfforce is given by some local terms which are relatively easy to calculate, plus a 'tail' term which, in the case of a scalar charge $q$ following a worldline $z(\tau)$, is given by

$$
q^{2} \int_{-\infty}^{\tau^{-}} \nabla_{\mu} G_{r e t}\left(z(\tau), z\left(\tau^{\prime}\right)\right) d \tau^{\prime}
$$

where $G_{r e t}\left(x, x^{\prime}\right)$ is the 'retarded' Green function of the scalar field wave equation. As the 'tail' term depends on the entire past history of the particle's worldline, it is usually quite difficult to calculate and one has to do so numerically.

\section{Method of Matched Expansions}

We choose to use the method of Matched Expansions ${ }^{2}$ to calculate (1). In this method, the 'tail' integral is separated into two regimes:

(a) In the quasilocal regime, $\tau^{\prime} \in(\tau-\Delta \tau, \tau)$ for a certain 'small value' $\Delta \tau$, the Green function is calculated using the Hadamard form:

$$
G_{r e t}\left(x, x^{\prime}\right)=\theta_{-}\left(x, x^{\prime}\right)\left\{\Delta^{1 / 2}\left(x, x^{\prime}\right) \delta(\sigma)-V\left(x, x^{\prime}\right) \theta(-\sigma)\right\}
$$


where $\sigma$ is half the square of the geodesic distance between the spacetime points $x$ and $x^{\prime}$, the van Vleck determinant $\Delta\left(x, x^{\prime}\right)$ and $V\left(x, x^{\prime}\right)$ are two regular biscalars and $\theta_{-}\left(x, x^{\prime}\right)$ guarantees causality. Given the structure of (2) it is clear that only $V\left(x, x^{\prime}\right)$ will contribute to the 'tail' integral in the quasilocal regime. The Hadamard form is only valid for points 'nearby' - within a normal neighbourhood.

(b) In the distant past regime, $\tau^{\prime} \in(-\infty, \tau-\Delta \tau)$, the Green function can be calculated using a Fourier mode decomposition and deforming the frequency-integral into a contour in the complex frequency-plane.

\section{Singularity structure of the Green function}

We carried out ${ }^{3}$ the first succesful application of the method of Matched Expansions. With the future view of applying it to the case of a point mass moving in an astrophysically-relevant spacetime, we used a toy model where calculations are easier: a scalar charge moving in the Nariai spacetime (cartesian product of 2-dimensional de Sitter spacetime and the 2-sphere), in which the radial potential of Schwarzschild is replaced by the Pöschl-Teller potential.

In the quasilocal regime, some powerful methods have been developed ${ }^{4,5}$ for the calculation of $V\left(x, x^{\prime}\right)$ up to, and beyond, the normal neighbourhood.

In the Nariai spacetime, the 'retarded' Green function in the 'distant past' is completely given by the sum over its poles - the quasinormal modes. A study of this series beautifully uncovered - Fig 1- the singularity structure of the Green function: its singularities occur at the times it takes a null geodesic to travel between $x$ and $x^{\prime}$ (i.e., when $\sigma=0$ ); furthermore, the singularity structure follows a 4 -fold cycle: $\delta(\sigma), 1 /(\pi \sigma),-\delta(\sigma),-1 /(\pi \sigma), \delta(\sigma), \ldots$

An alternative, heuristic way for understanding how this structure arises is by use of the Feynman Green function, which has Hadamard form

$$
G_{F}\left(x, x^{\prime}\right)=\frac{i}{2 \pi}\left\{\frac{\Delta^{1 / 2}\left(x, x^{\prime}\right)}{\sigma+i \epsilon}+V\left(x, x^{\prime}\right) \ln (\sigma+i \epsilon)+W\left(x, x^{\prime}\right)\right\}
$$

where $W\left(x, x^{\prime}\right)$ is another regular bitensor and $\epsilon>0$. The 'retarded' Green function can be obtained as $G_{r e t}\left(x, x^{\prime}\right)=2 \theta_{-}\left(x, x^{\prime}\right) \lim _{\epsilon \rightarrow 0} \operatorname{Re}\left(G_{F}\left(x, x^{\prime}\right)\right)$. Although strictly speaking the Hadamard form is only valid within the normal neighbourhood, if we tentatively use it beyond we can obtain the van Vleck determinant from a transport equation past caustic points, where it diverges. One then finds that $\Delta^{1 / 2}\left(x, x^{\prime}\right)$ picks up a factor ' $-i$ ' everytime a null geodesic crosses a caustic. From the property $\lim _{\epsilon \rightarrow 0^{+}} i /(\sigma+i \epsilon)=\pi \delta(\sigma)+i / \sigma$, one can see how the 4-fold cycle arises. This factor seems to be typical of the $\mathbb{S}^{2}$ topology and, although it is known in other fields of Science, in General Relativity it has only been previously observed by Prof. Ori, ${ }^{6}$ to the best of our knowledge.

We were able to successfully match the quasilocal and 'distant past' contributions to the 'retarded' Green function - Fig 2(a). The calculation of the 'retarded' Green function over the whole past of the particle's worldline enabled us to calculate

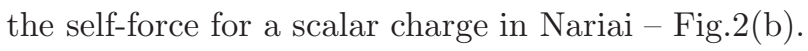



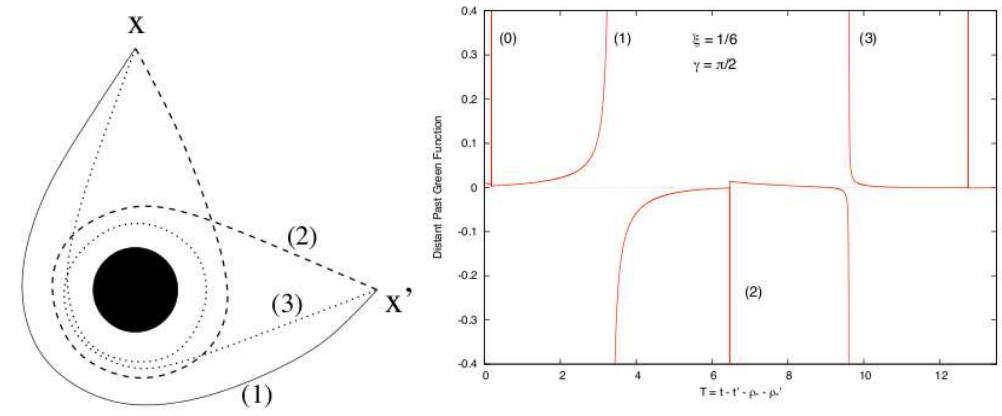

Fig. 1. (a) Null geodesics orbiting the unstable photon orbit of the background. (b) 'Retarded' Green function as a function of the proper time of the static scalar charge in Nariai. There is a 1-to-1 correspondence between the geodesics and the times when the Green function becomes singular, with a four-fold structure: $\delta(\sigma), \frac{1}{\pi \sigma},-\delta(\sigma),-\frac{1}{\pi \sigma}, \delta(\sigma), \ldots$
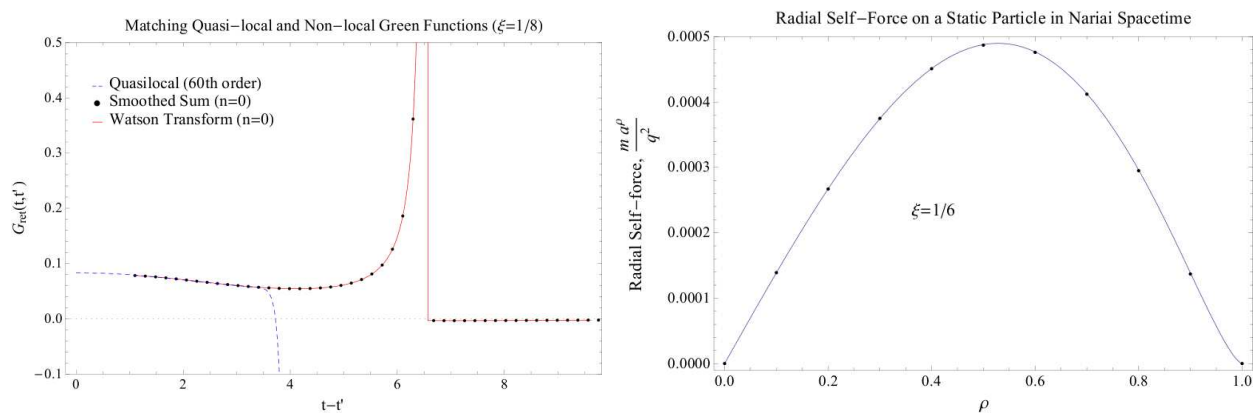

Fig. 2. (a) Matching of the 'quasilocal' and 'distant past' contributions as a function of time, (b) self-force for a static scalar charge in the Nariai spacetime as a function of the radius.

\section{Acknowledgements}

M.C. and B.W. are supported by the Irish Research Council for Science, Engineering and Technology.

\section{References}

1. E. Poisson, Living Rev. Relativity 7, p. 6 (2004).

2. E. Poisson and A. G. Wiseman, Suggestion at the 1st Capra ranch meeting on radiation reaction (1998).

3. M. Casals, S. Dolan, A. C. Ottewill and B. Wardell, Phys. Rev. D79, p. 124043 (2009).

4. M. Casals, S. Dolan, A. C. Ottewill and B. Wardell, Phys. Rev. D79, p. 124044 (2009).

5. A. C. Ottewill and B. Wardell (2009), arXiv:0906.0005.

6. A. Ori, private communication (2008) and report (2009) available at http://physics.technion.ac.il/ amos/acoustic.pdf 